\title{
Benjamin LEMOINE, L'Ordre de la dette
}

\section{Pierre de Saint-Phalle}

\section{OpenEdition \\ Journals}

Édition électronique

URL : http://journals.openedition.org/ress/3545

DOI : 10.4000/ress.3545

ISBN : 1663-4446

ISSN : 1663-4446

Éditeur

Librairie Droz

Édition imprimée

Date de publication : 30 novembre 2016

Pagination : 298-301

ISSN : 0048-8046

Référence électronique

Pierre de Saint-Phalle, "Benjamin LEMOINE, L'Ordre de la dette », Revue européenne des sciences sociales [En ligne], 54-2 | 2016, mis en ligne le 14 juillet 2016, consulté le 25 septembre 2020. URL http://journals.openedition.org/ress/3545; DOI : https://doi.org/10.4000/ress.3545

Ce document a été généré automatiquement le 25 septembre 2020

(C) Librairie Droz 


\title{
Benjamin LEMOINE, L'Ordre de la dette
}

\author{
Pierre de Saint-Phalle
}

\section{RÉFÉRENCE}

Benjamin LEMOINE, 2016, L'Ordre de la dette, Paris, La Découverte, 304 p.

1 Benjamin Lemoine est un spécialiste dans l'histoire contemporaine de la dette publique française, développant par ses travaux une analyse à la fois historique, sociologique et technique. L'ouvrage dont il est ici question est tiré de sa thèse de doctorat primée par l'Association française de science politique, Les Valeurs de la dette, l'État à l'épreuve de la dette publique dirigée par Michel Callon et Yannick Barthe. L'auteur, en retraçant les évolutions de la dette publique, cherche à repenser la nature de la dette et à comprendre son influence multiforme à la fois sur l'action de l'État et sur le débat public.

2 Préfacé par André Orléan, l'ouvrage s'inscrit dans une approche institutionnaliste du fait économique, à la frontière entre sociologie et économie. La réflexion menée porte sur les relations concrètes entre États et marchés financiers entraînant un phénomène de financiarisation de la valeur de l'État.

3 La dette de marché est un élément historiquement construit qui a des effets sur les politiques publiques possibles. L'ambition de l'ouvrage est de montrer qu'en quatre décennies, une architecture financière liant l'État français et les marchés financiers fut pensée, organisée et finalement considérée comme naturelle. La dette publique est un phénomène complexe, objet du débat public au moment des campagnes électorales ou des crises financières, mais qui disparaît de l'horizon médiatique entre-temps. Benjamin Lemoine a retranscrit étape par étape son évolution en tant que produit financier et politique, à la fois lors des coups de projecteurs médiatiques et lors des négociations feutrées au sein des ministères. 
Cette évolution a signifié le passage progressif d'un endettement contrôlé par l'État dans les années 1960 à un endettement négocié sur les marchés financiers dans les années 1980. La dette publique, aujourd'hui contrat de créance qui s'échange sur les marchés financiers, n'a pas toujours eu cette forme ni cette fonction. Le niveau d'endettement actuel, dépassant les $100 \%$ du PIB pour de nombreux pays dont la France, est généralement considéré comme un danger, le présage d'une possible catastrophe. Il est tout à fait étonnant, rétrospectivement, de lire l'enthousiasme dont témoignaient les acteurs procédant à la mise en marché de la dette publique, système présenté entre les années 1980 comme une chance pour l'État et pour les contribuables : se mettre en concurrence vis-à-vis des autres États devaient permettre une amélioration de l'usage des finances publiques, par le respect d'une discipline nouvelle issue du marché.

Benjamin Lemoine écrit un récit généalogique au sein duquel sont remises dans leurs contextes politiques autant les évolutions techniques (comme l'informatisation, le développement de nouveaux types de contrats de créances indexés sur l'inflation ou l'harmonisation des instruments de comptabilité internationales) que les réformes institutionnelles (comme le passage du circuit du trésor à l'adjudication, la création de l'agence France Trésor). Par ce récit, le lecteur assiste, pas à pas, à chaque étape de l'évolution complexe des mécanismes d'endettement public de l'État français et à leur incidence sur la façon dont les engagements de l'État sont hiérarchisés. Ces dispositifs techniques «modernes» de financement public entraînent simultanément une certaine évolution de l'État.

6 Selon Benjamin Lemoine, la dette de marché n'est pas un objet technique et neutre, mais le fruit d'une certaine vision de l'État, de ses missions, de sa valeur et de ses engagements. L'ouvrage porte sur un objet double : à la fois la matérialité de la dette (comment ont évolué techniquement l'endettement et les différents types de contrats de créances) et l'incidence de la dette sur l'action de l'État (l'évolutions des relations entre le Trésor, le gouvernement, les institutions internationales et les agences de notation).

7 Émaillé de témoignages d'acteurs de premiers plans, l'auteur a pu rencontrer hautfonctionnaires, banquiers et journalistes qui éclairent les réformes, rapports et débats institutionnels parfois menés en coulisses. Le livre est divisé en deux parties, chacune composée de 7 chapitres traitant les évènements de façon chronologique.

8 La première partie est le récit historique du passage du « circuit du Trésor », modalité d'endettement qui voit une domination de l'État vis-à-vis des institutions privées, au financement dit par adjudication dans les années 1980. L'État est toujours considéré comme souverain, mais les acteurs financiers nationaux privés sont peu à peu compris comme des partenaires ou entités à protéger voire à avantager. Petit à petit ce sont les finances publiques qui doivent être attrayantes, les indicateurs macroéconomiques (comme le chômage de masse faisant pression à la baisse sur les salaires) sont utilisés comme « arguments de vente » de la dette comme créance peu risquée.

9 Tout au long de ces premiers chapitres, l'auteur montre comment une certaine orthodoxie financière se focalisant sur la lutte contre l'inflation (doctrine défendue traditionnellement par le Trésor) va peu à peu s'imposer dans les différentes institutions. L'arrivée de la gauche au pouvoir en 1981 n'y change rien, c'est même sous le gouvernement Bérégovoy qu'est organisée en pratique la mise en marché de la dette publique. 

budgétaire particulière que l'auteur nomme «l'ordre de la dette ». L'une des thèses de l'ouvrage est que la légitimité des politiques d'austérité serait inscrite au cœur des instruments de mesure de la comptabilité publique. Les politiques budgétaires dites d'« austérité » ont pour objectif prioritaire l'« assainissement » des comptes publics, un déficit maîtrisé qui permet alors d'emprunter à des taux acceptables sur les marchés obligataires.

11 L'auteur observe qu'il s'opère un redéploiement des compétences des serviteurs de l'État, organisant des stratégies financières de défense de l'intérêt public dans le cadre du marché. Les acteurs auraient voulu introduire "une dose de libéralisme" en disciplinant l'État par les taux d'emprunt sans imaginer la situation actuelle : « la survie de l'appareil public dans son entier est désormais conditionnée à l'accompagnement et au renfort des puissances financières privées » (p. 150-151).

12 La seconde partie du livre s'attache à décortiquer les enjeux liés à la question de la dette publique entre les décennies 1990 et 2000. Le traité de Maastricht, instaurant l'objectif de $3 \%$ de déficit public et de $60 \%$ du PIB d'endettement public, a voulu rendre légale l'obligation de contrôle strict de l'endettement. La politique monétaire de la BCE de lutte contre l'inflation a participé à la naturalisation de la dette de marché et de ses contraintes budgétaires inhérentes.

13 L'auteur souligne la différence importante entre dette publique brute (simple calcul des encours issu de contrat de créances) et dette publique nette (passif moins actifs). Le traité de Maastricht, pour établir le célèbre ratio dette/PIB, n'avait retenu que la dette brute et non la dette nette afin de pouvoir mettre tous les États sur un même plan de comparaison de leurs efforts budgétaires respectifs.

14 L'auteur présente le débat normatif existant quant au sens à donner à la notion de dette publique. Ce débat sur la nature de la dette, issu des modalités de calcul retenues comme valides, ressurgit au moment du rapport Pébereau de 2005. Résultat d'une commission qui se voulait apolitique et non-partisane, ce rapport a, selon l'auteur, participé à la «dramaturgie de la dette publique ». Dans le débat public, la question de la dette est devenue un enjeu majeur teinté de «catastrophisme». La lutte contre sa croissance s'inscrit depuis lors comme un prérequis à toute position publique légitime sur la réforme des finances de l'État notamment pour l'élection présidentielle de 2007.

Benjamin Lemoine rappelle alors le lien entre le débat sur la réforme des retraites et le dispositif de dette publique. L'engagement pris par l'État à assurer le paiement des retraites est confronté à l'obligation de maintenir des critères budgétaires conforme à l'«orthodoxie financière » visant in fine à obtenir sur les marchés des taux d'emprunt faibles.

16 Comment inscrire les retraites des fonctionnaires dans le bilan de l'État? Cette question est, selon les témoignages recueillis, au cœur des débats de la commission Pébereau. Les retraites sont considérées comme des dettes implicites et flexibles, car il est politiquement possible de les modifier. Les contrats de créances sur les marchés sont des dettes explicites et rigides, il est déclaré impensable de les renégocier.

17 Benjamin Lemoine, en tirant le fil de la dette publique, cherche à écrire une certaine histoire contemporaine de l'absence d'horizon politique. La naturalisation des « bonnes gouvernances " proviendrait des outils de mesure qui mettraient davantage en une certaine forme les réalités budgétaires qu'ils ne les décriraient objectivement. C'est à

Revue européenne des sciences sociales, 54-2 | 2016 
une évolution de la valeur de l'État, par l'intégration de sa dette aux marchés financiers, à laquelle nous aurions assisté durant les quarante dernières années. Réorganiser les finances publiques pour permettre un endettement acceptable sur les marchés, au détriment des dettes sociales, aboutit à une transformation des priorités de la puissance publique. Sous l'apparence d'une allocation des ressources toujours plus efficiente, nous pourrions perdre notre identité politique par une évolution qui a les apparences de la nécessité. L'analyse historique permet, à cet égard, de se délier d'une telle nécessité pour voir toute la contingence de notre situation institutionnelle et économique.

Cet ouvrage, bien que fortement descriptif, a pour but de participer à une approche critique de l'architecture institutionnelle et financière, telle qu'elle fut pensée et telle qu'elle fut mise en œuvre. La crise de 2008 n'a que partiellement eu pour résultat un renouvellement théorique (pourtant nécessaire) de l'approche des relations entre régulation publique et activité des marchés. Le phénomène de la dette de marché en est peut-être la raison principale. Ce livre est une tentative ambitieuse - et réussie - de saisir un phénomène complexe, souvent déclaré trop technique. Son sujet est la nature profonde de notre régime politique et son évolution non-apparente. Comme le déclare Benjamin Lemoine : « la sociologie politique des problèmes économiques peut jouer son rôle d'aiguillage critique, en indiquant les zones ou porter le fer, relancer la controverse là ou elle s'est éteinte, et exiger le débat démocratique là ou il a été verrouillé et exclu» (p. 296).

\section{AUTEURS}

\section{PIERRE DE SAINT-PHALLE}

IEPHI - Centre Walras-Pareto, Université de Lausanne 